\title{
On power values of pyramidal numbers, I
}

\author{
Andrej Dujella, Kálmán Győry and Ákos Pintér*
}

To Professor Andrzej Schinzel on his 75th birthday

\begin{abstract}
For $m \geq 3$, the $m$ th order pyramidal number is defined by $x(x+$ $1)((m-2) x+5-m) / 6$. These combinatorial numbers play an important role in number theory and discrete mathematics. For $m=3$ and 4 , all the power values of these polynomials have been already found. In the present paper the authors deal with square values for larger $m$. All integer solutions of the corresponding elliptic curves are given for $3 \leq m \leq 100, m \neq 5$ (Proposition 1 ). The main result is the resolution, for a conjecturally infinite sequence of integers $m$, of the corresponding elliptic diophantine equation (Theorem 2).
\end{abstract}

2010 AMS Subject Classification: primary 11D25, 11D99; secondary 11Y50

Keywords: pyramidal numbers, square values, elliptic curves, integer points

\section{Introduction}

Let $m$ be a fixed integer with $m \geq 3$ and denote by

$$
\operatorname{Pyr}_{m}(x)=\frac{1}{6} x(x+1)((m-2) x+5-m)
$$

the $m$ th order pyramidal number. These combinatorial numbers play an important role in number theoory and discrete mathematics. They have

${ }^{*}$ Research supported in part by the Ministry of Science, Education and Sports, Republic of Croatia, grant 037-0372781-2821 (A. D.), the Hungarian-Croatian bilateral project Number theory and cryptography (A. D., K. Gy., Á. P.), the Hungarian Academy of Sciences (K. Gy., Á. P.), OTKA grants T67580, K75566, K100339 (K. Gy., Á. P.) and NK101680 (Á. P.), János Bolyai Fellowship (Á. P.) and the TÁMOP 4.2.1./B-09/1/KONV-20100007 project implemented through the New Hungary Development Plan co-financed by the European Social Fund, and the European Regional Development Fund (Á. P.). 
several interesting properties; see e. g. Dickson [9] and Conway and Guy [7]. In the classical cases $m=3$ and $m=4$, we have

$$
\operatorname{Pyr}_{3}(x)=\frac{1}{6} x(x+1)(x+2)=\left(\begin{array}{c}
x+2 \\
3
\end{array}\right)
$$

and

$$
\operatorname{Pyr}_{4}(x)=\frac{1}{6} x(x+1)(2 x+1)=1^{2}+2^{2}+\ldots+x^{2} .
$$

These numbers are related by $4 \mathrm{Pyr}_{4}(x)=\mathrm{Pyr}_{3}(2 x)$.

It was an old and hard problem to find all power values of the above polynomials of third degree. The corresponding equation has been resolved for squares by Watson [26], and for arbitrary powers by Györy [16], and Bennett, Györy and Pintér [5], respectively. For $x>2$, the only square values are given by $\left(\begin{array}{c}48+2 \\ 3\end{array}\right)=140^{2}$ and $1^{2}+2^{2}+\ldots+24^{2}=70^{2}$, respectively.

The purpose of this paper is to study the power values of pyramidal numbers of higher order, that is to extend the results mentioned above for larger values of $m$. In Part I we deal with squares values. The problem is then equivalent to finding the integer points on the elliptic curves

$$
E_{m}: \quad y^{2}=\frac{1}{6} x(x+1)((m-2) x+5-m)
$$

for positive integers $m \geq 3, m \neq 5$. For this reason, we exclude the case $m=5$ throughout the paper. For a fixed $m$, it is rather straightforward to determine all the integer points on $E_{m}$. For $3 \leq m \leq 100, m \neq 5$, we give all integer solutions of (1); cf. Proposition 1. The main result of our paper is the resolution, for a conjecturally infinite sequence of integers $m$, of the elliptic diophantine equation (1); cf. Theorem 2. In the forthcoming second part of this paper some explicit results on the higher power values of $\operatorname{Pyr}_{m}(x)$ will be presented. In that case the proofs require totally different methods.

\section{Preliminary observations and calculations}

By substitution $X=6(m-2) x, Y=36(m-2) y$, we obtain from (1) the following Weierstrass form:

$$
E_{m}^{\prime}: \quad Y^{2}=X^{3}+18 X^{2}+36(m-2)(5-m) X .
$$

There are three rational points on $E_{m}$ of order 2, namely

$$
A=(0,0), \quad B=(-1,0), \quad C=\left(\frac{m-5}{m-2}, 0\right),
$$


and also another obvious rational point $P=(1,1)$. It is easy to show, using Lutz-Nagell theorem, that $P$ cannot be of finite order. Namely, the point $2 P^{\prime}$ on $E_{m}^{\prime}$ does not have integer coordinates (the first coordinate is $\left.m^{2}-7 m+\frac{49}{4}\right)$.

Furthermore, we have

$$
\begin{gathered}
P+A=\left(-\frac{m-5}{m-2}, \frac{m-5}{m-2}\right), P+B=\left(-\frac{3 m}{2(m-2)},-\frac{2 m-7}{4(m-2)}\right), \\
P+C=\left(\frac{2 m-10}{3}, \frac{(m-5)(2 m-7)}{9}\right) .
\end{gathered}
$$

Therefore, we have always the following integer points on $E_{m}$ :

$$
(0,0),(-1,0),(1,1),
$$

and if $m \equiv 2 \quad(\bmod 3)$, then also $\left(\frac{2 m-10}{3}, \frac{(m-5)(2 m-7)}{9}\right)$.

Using the subroutine IntegralPoints of the program package MAGMA and the subroutine integral_points of the program package SAGE, we were able to find all integer points on $E_{m}$ for all $m \leq 100$. These subroutines use the elliptic logarithm method (introduced in [14] and [24], see also [23, 6]) for solving elliptic equations.

Proposition 1 Apart from the points listed in (3) and (4), in the range $3 \leq m \leq 100, m \neq 5$, all integer points on $E_{m}$ are:

$$
\begin{gathered}
(m, x, y)=(3,-2,0),(3,2,2),(3,48,140),(4,24,70),(7,6,14),(7,49,315), \\
(11,1681,84419),(13,24,160),(15,2,4),(15,242,5544),(16,49,525), \\
(20,49,595),(24,2,5),(24,1681,131979),(28,23,230),(29,8,48),(33,7,42), \\
(35,2,6),(35,49,805),(41,4,20),(41,49,875),(45,120,3520),(48,2,7), \\
(52,96,2716),(53,1681,200941),(62,49,1085),(63,2,8),(63,16,204), \\
\quad(68,24,390),(68,343,21070),(68,57121,45278311),(70,6,49), \\
(70,49,1155),(73,833,82705),(74,8,78),(75,10,110),(76,289,17255), \\
(76,3479,720650),(80,2,9),(80,1681,248501),(89,7,70),(91,4,30), \\
(91,6,56),(97,49,1365),(98,8,90),(99,2,10),(99,57121,54891369) .
\end{gathered}
$$

This provides all square values of pyramidal numbers for $3 \leq m \leq$ $100, m \neq 5$. In particular, it gives the square values of $\operatorname{Pyr}_{3}(x)$ and $\operatorname{Pyr}_{4}(x)$ mentioned in the Introduction. 
Notice that many integer points on $E_{m}$ have $x$-coordinate 2 . Indeed, for any $m$ of the form $y^{2}-1,(m, x, y)=\left(y^{2}-1,2, y\right)$ is an integer point on $E_{m}$. Further, there are several points on $E_{m}$ with $x$-coordinates 49 and 1681 . We will give an explanation for this fact later.

It is not hard to show, using $[20$, Main Theorem 1$]$, that $E_{m}(\mathbb{Q})_{\text {tors }} \simeq$ $\mathbb{Z} / 2 \mathbb{Z} \oplus \mathbb{Z} / 2 \mathbb{Z}$. However, it is much harder to predict the behavior of the ranks of $E_{m}(\mathbb{Q})$. In what follows, we deal with the case $\operatorname{rank}\left(E_{m}(\mathbb{Q})\right)=1$. We first make some preliminary remarks.

Let us consider the elliptic curve $\mathcal{E}$ over $\mathbb{Q}(T)$ :

$$
\mathcal{E}: y^{2}=x^{3}+18 x^{2}+36(T-2)(5-T) x .
$$

The discriminant of $\mathcal{E}$ is $D=746496(T-5)^{2}(2 T-7)^{2}(T-2)^{2}$. We can compute $\operatorname{rank}_{\mathbb{C}(T)} \mathcal{E}$ using Shioda's formula [21, Corollary 5.3]:

$$
\operatorname{rank}_{\mathbb{C}(T)} \mathcal{E}=\operatorname{rank} N S(\mathcal{E}, \mathbb{C})-2-\sum_{s}\left(m_{s}-1\right) .
$$

Here $N S(\mathcal{E}, \mathbb{C})$ is the Néron-Severi group of $\mathcal{E}$ over $\mathbb{C}$, and the sum ranges over all singular fibres of the pencil $\mathcal{E}_{t}$, with $m_{s}$ the number of irreducible components of the fibre, which can be easily determined from Kodaira types of singular fibres (in our case they are $\mathrm{I}_{0}^{*}, \mathrm{I}_{2}, \mathrm{I}_{2}, \mathrm{I}_{2}$ ). Since $\mathcal{E}$ is a rational surface, we have $\operatorname{rank} N S(\mathcal{E}, \mathbb{C})=10$. Therefore, we have

$$
\operatorname{rank}_{\mathbb{C}(T)} \mathcal{E}=10-2-4-1-1-1=1 .
$$

Since we already know that $\operatorname{rank}_{\mathbb{Q}(T)} \mathcal{E} \geq 1$ (because the point $P$ is of infinite order), we conclude that $\operatorname{rank}_{\mathbb{Q}(T)} \mathcal{E}=1$.

Standard conjectures on the distribution of ranks in families of elliptic curves suggest that $50 \%$ of curves in the family $E_{m}$ have rank 1, while $50 \%$ of curves have rank 2. We have computed, using Mwrank [8], the ranks for $m \in\{3,4,6,7, \ldots, 200\}$ and obtained the following distribution of ranks: 80 cases of rank 1, 95 cases of rank 2, 20 cases of rank 3 and 2 cases of rank 4. For larger values of $m$, we found also several curves with rank 5,6 and 7. Using standard sieving methods (see e.g. [11]), we were able to find examples with rank equal to 8 for $m=8704960$ and $m=11007950$.

\section{A family of elliptic curves}

In this section we obtain some results on integers points on curves $E_{m}$ under the assumption that $\operatorname{rank}\left(E_{m}(\mathbb{Q})\right)=1$. The above discussion shows that this assumption is not unrealistic. We will follow the strategy from [10]. 
First we need the result that $P, P+A, P+B, P+C \notin 2 E_{n}(\mathbb{Q})$. This follows easily, by applying 2-descent Proposition [17, Theorem 4.3] to points $P^{\prime}, P^{\prime}+A^{\prime}, P^{\prime}+B^{\prime}, P^{\prime}+C^{\prime}$ on the curve $E_{m}^{\prime}$. Assume that $E_{m}(\mathbb{Q}) / E_{n}(\mathbb{Q})_{\text {tors }}=$ $\langle U\rangle$. Let $X \in E_{m}(\mathbb{Q})$. Then we can represent $X$ in the form $X=k U+T$, where $k$ is an integer and $T$ is a torsion point, i.e. $T \in\{\mathcal{O}, A, B, C\}$. Also, $P=k_{P} U+T_{P}$ for an integer $k_{P}$ and a torsion point $T_{P}$. We know that $k_{P}$ is odd. Hence, $U \equiv P+T_{P}\left(\bmod 2 E_{m}(\mathbb{Q})\right)$. Therefore, we have $X \equiv X_{0}$ $\left(\bmod 2 E_{m}(\mathbb{Q})\right)$, where $X_{0} \in \mathcal{S}=\{\mathcal{O}, A, B, C, P, P+A, P+B, P+C\}$.

Let $\{a, b, c\}=\{0,6 m-12,30-6 m\}$. By [17, Proposition 4.6], the function $\varphi: E_{m}^{\prime}(\mathbb{Q}) \rightarrow \mathbb{Q}^{*} / \mathbb{Q}^{* 2}$ defined by

$$
\varphi(X)= \begin{cases}(x+a) \mathbb{Q}^{* 2} & \text { if } X=(x, y) \neq \mathcal{O},(-a, 0) \\ (b-a)(c-a) \mathbb{Q}^{* 2} & \text { if } X=(-a, 0) \\ \mathbb{Q}^{* 2} & \text { if } X=\mathcal{O}\end{cases}
$$

is a group homomorphism. Therefore, in order to find all integer points on $E_{m}$, it suffices to solve in integers all systems of equations of the form

$$
x=\alpha \square, \quad x+1=\beta \square, \quad(m-2) x+5-m=\gamma \square
$$

where for $X_{0}=(6(m-2) s, 36(m-2) t) \in \mathcal{S}$, the numbers $\alpha, \beta, \gamma$ are defined by $\alpha=s, \beta=s+1, \gamma=(m-2) s+5-m$ if all of these three expressions are nonzero, and if e.g. $s=0$ then we define $\alpha=\beta \gamma$. Here $\square$ denotes the square of a rational number.

Consider now (5) for the possible choices of $X_{0}$.

1) $X_{0}=\mathcal{O}$. The system of equations (5) becomes

$$
\begin{aligned}
x & =6(m-2) \square, \\
x+1 & =6(m-2) \square, \\
(m-2) x+5-m & =6 \square .
\end{aligned}
$$

Since $\operatorname{gcd}(x, x+1)=1$, we must have that $m-2=6 \square$. This gives $x=$ and $x+1=\square$, which leads to a contradiction.

2) $X_{0}=A$. Now the system of equations (5) becomes

$$
\begin{aligned}
x & =-6(m-5) \square, \\
x+1 & =\square, \\
(m-2) x+5-m & =-(m-5) \square .
\end{aligned}
$$

One solution of this system of equations is $x=0$. For $m \geq 6$ there are no other solutions since $x$ and $x+1$ cannot be of opposite sign. Both 
cases $m=3$ and $m=4$ lead to the system of Pell equation $v^{2}-6 u^{2}=1$, $w^{2}-12 u^{2}=1$, which has the unique solution $u=2, v=5, w=7$ (see [1]). This gives the additional solution $x=48$ for $m=3$, and $x=24$ for $m=4$.

3) $X_{0}=B$. We obtain the system of equations

$$
\begin{aligned}
x & =-\square, \\
x+1 & =6(2 m-7) \square, \\
(m-2) x+5-m & =-(2 m-7) \square,
\end{aligned}
$$

which obviously has the only solution $x=-1$, since otherwise for $m \geq 4$ the integers $x$ and $x+1$ would be of opposite sign (for $m=3$ the same holds for $x$ and $x+2$ ).

4) $X_{0}=C$. In this case we obtain the system of equations

$$
\begin{aligned}
x & =(m-2)(m-5) \square, \\
x+1 & =(m-2)(2 m-7) \square, \\
(m-2) x+5-m & =6(m-5)(2 m-7) \square .
\end{aligned}
$$

This system may have a solution only if $m-2=k^{2}$ or $m-2=3 k^{2}$ for an integer $k$, since otherwise the square-free part of $m-2$ or $(m-2) / 3$ would divide both $x$ and $x+1$. For $m=k^{2}+2$ and $m=3 k^{2}+2$ we were not able to solve the system of equations unconditionally. It is easy to check that for $m=1^{2}+2=3$ the only solution is $x=-2$.

5) $X_{0}=P$. In this case we have the system of equations

$$
\begin{aligned}
x & =\square, \\
x+1 & =2 \square, \\
(m-2) x+5-m & =3 \square
\end{aligned}
$$

which has an obvious solution $x=1$. For certain integers $m$ there can be additional solutions. From $x=u^{2}, x+1=2 v^{2}$, we find the possibilities for $x$, namely, $x=7^{2}, 41^{2}, 239^{2}, \ldots$ Inserting this in the third equation of the system of equations, we obtain a condition for $m$. E.g. for $x=49$, we obtain $16 m=w^{2}+31$, which is satisfied for $m=7,16,20,35,41,62$, $70,97,107,140,152,191, \ldots$, while for $x=1681$ we obtain $560 m=w^{2}+1119$, which is satisfied for $m=11,24,53,80,104,141,143,186, \ldots$. Note that for $m=30890$ we have both additional solutions $x=49$ and $x=1681$. Note that for the most of these values of $m$, the rank of $E_{m}$ is greater than 1 . The only known exception is $m=11$, where the rank is equal to 1 and $x(3 P)=1681$. Note also that for $m=3$ and $m=4$ the only solution of the above system is $x=1$, which follows from [4]. 
6) $X_{0}=P+A$. It is easy to see that the corresponding system of equations

$$
\begin{aligned}
x & =-(m-2)(m-5) \square, \\
x+1 & =3(m-2) \square, \\
(m-2) x+5-m & =-2(m-5) \square
\end{aligned}
$$

has no solution for $m \geq 6$, because $x$ and $x+1$ have the same sign. There is no solution also for $m=4$, while the only solution for $m=3$ is $x=2$.

7) $X_{0}=P+B$. Then the system of equations is

$$
\begin{aligned}
x & =-6(m-2) \square, \\
x+1 & =2(m-2)(2 m-7) \square, \\
(m-2) x+5-m & =-2(2 m-7) \square
\end{aligned}
$$

which is clearly unsolvable. Again, it is enough to use the fact that integers $x$ and $x+1$ have the same sign.

8) $X_{0}=P+C$. In this case we have the system of equations

$$
\begin{aligned}
x & =6(m-5) \square, \\
x+1 & =3(2 m-7) \square, \\
(m-2) x+5-m & =3(m-5)(2 m-7) \square .
\end{aligned}
$$

This system has an obvious rational solution $x=\frac{2}{3}(m-5)$. Therefore, if $m \equiv 2(\bmod 3)$ this gives an integer solution of the system of equations. On the other hand, it is clear that if $m \not \equiv 2(\bmod 3)$, then there is no integer solution, since $x$ and $x+1$ cannot be both divisible by 3 .

Thus, let us consider the above system of equations for $m=3 k+2$. Then we have

$$
x=2(k-1) u^{2}, \quad x+1=(2 k-1) v^{2}, \quad k x-k+1=(k-1)(2 k-1) w^{2} .
$$

It seems that the only solution of $(6)$ is $x=2 k-2=\frac{2}{3}(m-5)$. However, we are able to prove this statement only under the additional assumption that the integers $k-1$ and $2 k-1$ are square-free. It is well known that there exist infinitely many positive integers $k$ with this property. These assumptions imply that $u, v, w$ in (6) are integers, and we obtain the system of Pellian equations

$$
\begin{aligned}
2 k u^{2}-(2 k-1) w^{2} & =1 \\
k v^{2}-(k-1) w^{2} & =1 .
\end{aligned}
$$


We have $v=p_{j}=q_{l}$, where the sequences $\left(p_{j}\right)$ and $\left(q_{l}\right)$ are defined by

$$
\begin{gathered}
p_{0}=1, \quad p_{1}=8 k-1, \quad p_{j+2}=(8 k-2) p_{j+1}-p_{j}, \\
q_{0}=1, \quad q_{1}=4 k-1, \quad q_{l+2}=(4 k-2) q_{l+1}-q_{l} .
\end{gathered}
$$

Using the method described in [12], we will show that the only solution of the equation $p_{j}=q_{l}$ is $j=l=0$, which corresponds to $u=v=w=1$ and $x=2 k-2$. In fact, the system of equations solved in [12] is very similar to (7) and (8), so we will omit the details of the proof and give just a short overview. Using the congruence relations $p_{j} \equiv(-1)^{j-1}(4 j(j+1) k-1)\left(\bmod 64 k^{2}\right)$ and $q_{l} \equiv(-1)^{l-1}(2 l(l+1)-1)\left(\bmod 16 k^{2}\right)$, we obtain a lower bound for the solutions: $l \geq j \geq 2 k-0.5$. Comparing it with the upper bound which follows from Bennett's theorem on simultaneous approximations [3] we finish the proof for sufficiently large values of $m$. For small $m$, the statement can be proved by Baker-Davenport reduction, see [2] or [13].

We have solved completely the systems of equations corresponding to the points $\mathcal{O}, A, B, P+A, P+B$, while the systems of equations corresponding to $C, P, P+C$ are solved only for values of parameter $m$ satisfying certain conditions. We will solve completely all eight systems of equations, i.e. we will find all integer points for a (conjecturally infinite) subfamily of $E_{m}$. Let $m=3 k^{4}+2$. The family $E_{3 k^{4}+2}$ has generic rank 1 over $\mathbb{Q}(k)$, which can be proved by 2 -descent method, as in [10], by finding suitable specialization with rank 1 (e.g. $k=60)$.

Theorem 2 Let $k$ be positive integer such that $2 k^{4}-1$ is square-free, while $k^{4}-1=2^{s} t$ for $s \geq 0$ and a square-free odd number $t$. Let $m=3 k^{4}+2$. If $\operatorname{rank}\left(E_{m}(\mathbb{Q})\right)=1$, then all integer points on $E_{m}$ are:

$$
(0,0),(-1,0),(1,1),\left(2 k^{4}-2,2 k^{8}-3 k^{4}+1\right) .
$$

This gives all square values of the pyramidal numbers for the numbers $m$ under consideration.

The conditions of Theorem 2 are satisfied for $k=2,3,4,9,16,25,47,59$, $60,65,78,85,87, \ldots$ The first few corresponding $m$ 's are $m=50,245,770$, 19685,196610 . The conditions concerning $k^{4}-1$ and $2 k^{4}-1$ are conjecturally satisfied for infinitely many positive integers $k$, see [15].

Proof of Theorem 2. Having the results obtained in the cases 1) to 8), it suffices to solve the corresponding systems of equations for the points $C, P$ and $P+C$ only. 
Because of the special form of $m$, we have to consider the system of equations corresponding to the point $C$ :

$$
\begin{aligned}
x & =\left(k^{4}-1\right) \square, \\
x+1 & =\left(2 k^{4}-1\right) \square, \\
k^{4} x+1-k^{4} & =2\left(k^{4}-1\right)\left(2 k^{4}-1\right) \square .
\end{aligned}
$$

The third equation shows that there is no solution if $k$ is even. For odd $k$, from the first and third equations we obtain

$$
k^{4} u^{2}-2\left(2 k^{4}-1\right) w^{2}=2^{s} \text { or } 2^{s-1},
$$

according as $s$ is even or odd. Since $k$ is odd, $u$ must be even, and repeating this argument we arrive at the equation

$$
k^{4} u^{\prime 2}-2\left(2 k^{4}-1\right) w^{\prime 2}=1 .
$$

The fundamental solution of the Pell equation $U^{2}-2\left(2 k^{4}-1\right) W^{2}=1$ is $U_{0}=2 k^{4}-1, W_{0}=2 k^{2}$. Since $W_{0} \mid W$, we conclude that $k \mid w^{\prime}$ in (9), which is impossible for $k>1$.

Consider now the system of equations corresponding to the point $P$. From the first and third equations we obtain

$$
w^{2}-\left(u^{2}-1\right) k^{4}=1,
$$

which we consider as an equation in unknowns $w$ and $k$. Here we may apply the result of Ljungrren [18, 25], which implies that if the equation (10) has two solutions, then $k^{2}=2 u$ or $k^{2}=8 u^{3}-4 u$. The first possibility implies that $u$ is even, which is in contradiction to

$$
u^{2}-2 v^{2}=-1 .
$$

The second possibility can occur only for $u=169$, which again contradicts (11). Hence, the only solution to the equation (10) is $w=u, k=1$.

There remains the seventh system of equations corresponding to the point $P+C$. If $k$ is even, then our assumption implies that $k^{4}-1$ is square-free, and we have already solved the system of equations under this assumption. For $k$ odd, we obtain the system of equations

$$
\begin{aligned}
2 k^{4} u^{2}-\left(2 k^{4}-1\right) w^{2} & =2^{s} \text { or } 2^{s-1} \\
k^{4} v^{2}-t w^{2} & =1
\end{aligned}
$$


which can be written in the form

$$
\begin{aligned}
2 k^{4} u^{2}-\left(2 k^{4}-1\right) w^{2} & =1 \\
k^{4} v^{2}-\left(k^{4}-1\right) w^{\prime 2} & =1 .
\end{aligned}
$$

This is a special case of the system of equations (7), (8). But we have already shown that its only solution is $v=0$, i.e. $x=2 k^{4}-2$.

Remark. Note that although we have followed the strategy from [10], there are some significant differences between the two families. The results of [10] suggest (see also [19]) that the number of integer points on the elliptic curves

$$
y^{2}=((k-1) x+1)((k+1) x+1)(4 k x+1)
$$

is independent of the value of $k$, in particular, that it does not depend on the rank of the curve. It is in big contrast with what is conjectured (and partly proved) for elliptic curves in Weierstrass form, where it is expected that the number of integer points grows exponentially with the rank (see [22]). For our family of curves, the dependence of the number of integer points on the rank of curve is not so clear, and we believe that this is a challenging question.

\section{References}

[1] W. A. Anglin, Simultaneous Pell equations, Math. Comp. 65 (1996), 355-359.

[2] A. Baker and H. Davenport, The equations $3 x^{2}-2=y^{2}$ and $8 x^{2}-7=z^{2}$, Quart. J. Math. Oxford Ser. (2) 20 (1969), 129-137.

[3] M. A. Bennett, On the number of solutions of simultaneous Pell equations, J. Reine Angew. Math. 498 (1998), 173-199.

[4] M. A. Bennett, On consecutive integers of the form $a x^{2}, b y^{2}$ and $c z^{2}$, Acta Arith. 88 (1999), 363-370.

[5] M. A. Bennett, K. Győry and Á. Pintér, On the diophantine equation $1^{k}+$ $2^{k}+\ldots+x^{k}=y^{n}$, Compositio Math., 140 (2004), 1417-1431.

[6] H. Cohen, Number Theory. Volume I: Tools and Diophantine Equations, Springer, New York, 2007.

[7] J. H. Conway and R. K. Guy, The Book of Numbers, Springer, New York, 1996.

[8] J. E. Cremona, Algorithms for Modular Elliptic Curves, Cambridge Univ. Press, 1997. 
[9] L. E. Dickson, History of the Theory of Numbers: Diophantine Analysis, Volume II, Chelsea Publ.,New York, 1971.

[10] A. Dujella, A parametric family of elliptic curves, Acta Arith. 94 (2000), 87101.

[11] A. Dujella, On Mordell-Weil groups of elliptic curves induced by Diophantine triples, Glas. Mat. Ser. III 42 (2007), 3-18.

[12] A. Dujella and B. Jadrijević, A parametric family of quartic Thue equations, Acta Arith. 101 (2002), 159-170.

[13] A. Dujella and A. Pethö, Generalization of a theorem of Baker and Davenport, Quart. J. Math. Oxford Ser. (2) (49) (1998), 291-306.

[14] J. Gebel, A. Pethő and H. G. Zimmer, Computing integral points on elliptic curves, Acta Arith. 68 (1994), 171-192.

[15] A. Granville, $A B C$ allows us to count squarefrees, Internat. Math. Res. Notices 1998, 991-1009.

[16] K. Györy, On the diophantine equation $\left(\begin{array}{l}n \\ k\end{array}\right)=x^{l}$, Acta Arith., 80 (1997), 289-295.

[17] A. Knapp, Elliptic Curves, Princeton Univ. Press, 1992.

[18] W. Ljunggren, Zur Theorie der Gleichung $x^{2}+1=D y^{4}$, Avh. Norsk. Vid. Akad. Oslo (1942), 1-27.

[19] F. Najman, Compact representation of quadratic integers and integer points on some elliptic curves, Rocky Mountain J. Math., 40 (2010), 1979-2002.

[20] K. Ono, Euler's concordant forms, Acta Arith. 78 (1996), 101-123.

[21] T. Shioda, On the Mordell - Weil lattices, Comment. Math. Univ. St. Pauli 39 (1990), 211-240.

[22] J. H. Silverman, The Arithmetic of Elliptic Curves, Springer-Verlag, New York, 1986.

[23] N. P. Smart, The Algorithmic Resolution of Diophantine Equations, Cambridge University Press, Cambridge, 1998.

[24] R. J. Stroeker and N. Tzanakis, Solving elliptic diophantine equations by estimating linear forms in elliptic logarithms, Acta Arith. 67 (1994), 177-196.

[25] P. G. Walsh, A note on a theorem of Ljunggren and the Diophantine equations $x^{2}-k x y^{2}+y^{4}=1,4$, Arch. Math. 72 (1999), 1-7. 
[26] G. N. Watson, The problem of the square pyramid, Messenger of Math. 48 (1918), 1-22.

\author{
Andrej Dujella \\ Department of Mathematics \\ University of Zagreb \\ Bijenička cesta 30 \\ 10000 Zagreb \\ CROATIA \\ e-mail: duje@math.hr
}

Kálmán Győry and Ákos Pintér

Institute of Mathematics and the Hungarian Academy of Sciences

University of Debrecen

Egyetem tér 1.

H-4032 Debrecen

HUNGARY

e-mail: gyory@science.unideb.hu, apinter@science.unideb.hu 